\title{
ON THE ARITHMETIC OF WEIGHT SPACES IN THE ROOT LATTICE
}

\author{
ROBERT W. DECKHART
}

(Communicated by Warren J. Wong)

\begin{abstract}
Using the notion of $i$-blocks in a weight space of the positive cone of the root lattice we obtain, after suitable reduction to nontrivial cases, a surprising strongly inductive formula for Kostant's partition function in terms of exactly 3 lower weight spaces. The proof is elementary, using arithmetic properties of $i$-blocks from [2], and avoiding any character formulas.
\end{abstract}

\section{INTRODUCTION AND APPLICATIONS}

Fix a base $B$ for a root system $R$ and use it to define the root lattice $\Lambda_{r}$ as the Z-linear combinations of the members of $B$, and $\Lambda_{r}^{+}$as the $\mathbf{Z}_{+}$linear combinations. For $\tau \in \Lambda_{r}^{+}$let $P(\tau)$ denote Kostant's partition function, the number of ways to write $\tau$ as a sum of positive roots. In [2] the Kostant cone of $\Lambda_{r}^{+}$, here denoted $\Lambda_{r}^{++}$, is introduced. A $\tau \in \Lambda_{r}^{+}$is in the Kostant cone if it can be written as a sum of nonsimple roots. There is a bracketing function $s$ from $\Lambda_{r}^{+}$to $\Lambda_{r}^{+}$which is idempotent, whose image is $\Lambda_{r}^{++}$, whose fixed points are precisely $\Lambda_{r}^{++}$, and is such that $P(s(\tau))=P(\tau)$ for all $\tau \in \Lambda_{r}^{+}$. So any calculation of $P(\tau)$ can be reduced to the Kostant cone. But there is little indication, so far, of how to compute $P(\tau)$ once in the Kostant cone.

For $\tau \in \Lambda_{r}^{+}$let $E_{+}(\tau)$ be the set of things that $P(\tau)$ counts. There are many useful ways to subdivide $E_{+}(\tau)$. The one used here is to partition $E_{+}(\tau)$ into $i$-blocks, $Q_{i}(j)$. In [2] there is a duality theorem relating pairs of $i$-blocks of $E_{+}(\tau)$. This result is a useful arithmetic version of the geometric fact that a reflection in a simple root $\alpha_{i}$ permutes the positive roots other than $\alpha_{i}$. We can describe this duality in terms of the root and weight coordinates of $\tau$.

Let $\tau \in \Lambda_{r}^{+}$. The root lattice is contained in the weight lattice $\Lambda$, i.e., the set of $\mathbf{Z}$-linear combinations of members of $D$, where $D$ is the set of fundamental dominant weights with respect to $B$. So we have $\tau=\sum r_{i} \alpha_{i}$, in terms of $\Lambda_{r}^{+}$, and $\tau=\sum w_{i} \lambda_{i}$, in terms of $\Lambda$. Fix the index $i$. The duality result states that if there is a nonnegative integer $j$ such that $j \leq r_{i}$ and $j \leq w_{i}$, then we have $\left|Q_{i}(j)\right|=\left|Q_{i}\left(w_{i}-j\right)\right|$. We are most concerned when $j=0$. For $\tau$ in the

Received by the editors April 11, 1988.

1980 Mathematics Subject Classification (1985 Revision). Primary 17B10, 22 E47. 
Kostant cone, $\left|Q_{i}(0)\right|>0$ for all $i$. There is always an index $i$ with $w_{i}>0$ for nonzero $\tau \in \Lambda_{r}^{++}$, thus giving a nontrivial $i$-block relation by duality.

This $i$-block relation yields our main result here, Theorem 5 , a strongly inductive formula for $P(\tau)$. Let $\tau \in \Lambda_{r}^{++}$with $\tau \neq 0$ and $\tau=\sum w_{i} \lambda_{i}$. For at least one $i, P(\tau)-P\left(\tau-\alpha_{i}\right)=P\left(\tau-w_{i} \alpha_{i}\right)-P\left(\tau-\left(w_{i}+1\right) \alpha_{i}\right)$ where $P(\tau)>P\left(\tau-\alpha_{i}\right) \geq P\left(\tau-w_{i} \alpha_{i}\right)>P\left(\tau-\left(w_{i}+1\right) \alpha_{i}\right)$.

There were two situations in the representation theory of semisimple Lie algebras in which the reduction of the calculation of $P(\tau)$ to those $\tau \in \Lambda_{r}^{++}$ was used with effect. First, from [2], let $L$ be a complex simple Lie algebra with root system $R$ and $V$ a finite dimensional $L$-module of highest weight $\lambda$. Let $m_{\lambda}(\tau)$ denote the multiplicity of the weight space of weight $\lambda-\tau$ in $V$, where $\tau \in \Lambda_{r}^{+}$. If $\lambda-\tau$ is a dominant weight, then $m_{\lambda}(\tau)=m_{\lambda}(s(\tau))$, mimicking the behavior of $P(\tau)$ in $\Lambda_{r}^{+}$. Of course this doesn't work for nondominant $\lambda-\tau$, else $V$ would be infinite dimensional.

In case $\tau$ is sufficiently close to $\lambda$, i.e., $\lambda=\sum m_{i} \lambda_{i}$ and $\tau=\sum r_{i} \alpha_{i}$ with $r_{i} \leq$ $m_{i}$ for all $i$, we have $P(\tau)=m_{\lambda}(\tau)$, so in this case we have, for nonzero $\tau \in$ $\Lambda_{r}^{++}$, that $m_{\lambda}(\tau)-m_{\lambda}\left(\tau-\alpha_{i}\right)=m_{\lambda}\left(\tau-w_{i} \alpha_{i}\right)-m_{\lambda}\left(\tau-\left(w_{i}+1\right) \alpha_{i}\right)$. This formula doesn't always hold for general $\tau \in \Lambda_{r}^{++}$with $\lambda-\tau$ dominant. There are, for example, one-dimensional dominant weight spaces in the Kostant cone, in which case this inductive formula, or any reasonable analog, will never work. But with a complete classification of these weight spaces, careful use of the operator $s$, and understanding of the behavior of $s$ near the walls of the dominant chamber, then it is very likely that an intuitive and extremely fast multiplicity formula, as compared to the very fast formulas of Moody and Patera [7], [8], see also Pianzola [9], will result. Also we get useful inequalities.

The second place where this reduction to the Kostant cone was used is in prime characteristic versions of these irreducible $L$-modules in the case of a $p$-restricted highest weight $\lambda$, see [3]. Modular maximal vectors, which would herald submodules mod- $p$, are to be found only in weight spaces of weight $\lambda-\tau$ for $\tau \in \Lambda_{r}^{++}$, adding a second condition to $p$-linkage, see Humphreys [6], for such vectors. Interestingly, the one-dimensional dominant weight spaces in the Kostant cone are related to the $p=2$ (and $p=3$ for $G_{2}$ ) modular maximal vectors.

Finding $p$-modular maximal vectors involves careful study of the relations between the weight spaces of $\tau \in \Lambda_{r}^{++}$and $\tau-\alpha_{i} \in \Lambda_{r}^{+}$, for all $\alpha_{i} \in B$. We need to know the multiplicity jumps for these, as well as how bases for these weight spaces are related. By starting with those $i$ such that our main formula holds we get a great head start on the construction of modular maximal vectors by looking at the $\tau-w_{i} \alpha_{i}$ and $\tau-\left(w_{i}+1\right) \alpha_{i}$ weight spaces, which are in inductive range. For indices $i$ such that Theorem 5 does not apply we have a certain regular degeneration. This will be discussed at length in part II of reference [4]. 
This paper is an expanded version of a letter to A. J. Coleman, in which I wanted to present a useful model for a "definitive" multiplicity formula.

We begin with a section reviewing some results of [2], with some notational changes.

\section{RoOT LATTICE FUNCTIONS}

Let $\mathbf{Z}$ denote the ring of integers and $\mathbf{Z}_{+}$the nonnegative integers. Let $R$ be reduced irreducible root system of rank $n$ with base $B=\left\{\alpha_{1}, \ldots, \alpha_{n}\right\}$ and positive roots $R^{+}=\left\{\beta_{1}, \ldots, \beta_{m}\right\}$, where $\beta_{1}=\alpha_{1}, \ldots, \beta_{n}=\alpha_{n}$. Let $D=\left\{\lambda_{1}, \ldots, \lambda_{n}\right\}$ be the fundamental dominant weights with respect to $B$. Let $\Lambda$ be the weight lattice and $\Lambda_{r}$ the root lattice, the sets of $\mathbf{Z}$-linear combinations of the members of $D$ and $B$ respectively, with $\Lambda^{+}$and $\Lambda_{r}^{+}$the $\mathbf{Z}_{+}$-linear combinations.

If $\tau \in \Lambda_{r}^{+}, \tau=\sum r_{i} \alpha_{i}$, then $\tau \in \Lambda$, say $\tau=\sum w_{i} \lambda_{i}$. We define $\Upsilon$, the root coordinate function, from $\Lambda_{r}^{+}$to $\mathbf{Z}_{+}^{n}, \Omega$, the weight coordinate function from $\Lambda_{r}^{+}$to $\mathbf{Z}^{n}$, and $\Gamma$, the capacity function from $\Lambda_{r}^{+}$to $\mathbf{Z}^{n}$ as follows: for $\tau \in \Lambda_{r}^{+}, \tau=\sum r_{i} \alpha_{i}=\sum w_{i} \lambda_{i}$, we set $\Upsilon(\tau)=\left(r_{1}, \ldots, r_{n}\right) \in \mathbf{Z}_{+}^{n}, \Omega(\tau)=$ $\left(w_{1}, \ldots, w_{n}\right) \in \mathbf{Z}^{n}$, and $\Gamma(\tau)=\left(g_{1}, \ldots, g_{n}\right) \in \mathbf{Z}^{n}$, where $g_{i}=r_{i}-w_{i}, 1 \leq$ $i \leq n$.

For $\tau \in \Lambda_{r}^{+}$let $E_{+}(\tau)$ denote the set of all expressions for $\tau$ as a $\mathbf{Z}_{+}$-sum of positive roots. The coordinates for a member of $E_{+}(\tau)$ would be in $\mathbf{Z}_{+}^{m}$. We define $P$, the root version of Kostant's partition function, from $\Lambda_{r}$ to $\mathbf{Z}_{+}$ as follows: for $\tau \in \Lambda_{r}^{+}, P(\tau)=\left|E_{+}(\tau)\right|$, and for $\tau \in \Lambda_{r}-\Lambda_{r}^{+}, P(\tau)=0$.

The Kostant cone of $\Lambda_{r}^{+}$, denoted $\Lambda_{r}^{++}$, is the set of $\tau \in \Lambda_{r}^{+}$such that $E_{+}(\tau)$ contains at least one member with no simple root summands. Thus $\tau$ is in the Kostant cone if we can write it as a sum, over $\mathbf{Z}_{+}$, of roots from $R^{+}-B$.

We define the function $s$ from $\Lambda_{r}^{+}$to $\Lambda_{r}^{+}$as follows: for $\tau \in \Lambda_{r}^{+}$with $\Upsilon(\tau)=\left(r_{1}, \ldots, r_{n}\right)$ and $\Gamma(\tau)=\left(g_{1}, \ldots, g_{n}\right)$ we set $s(\tau)=\sum s_{i} \alpha_{i}$ where, for $1 \leq i \leq n, s_{i}=\min \left\{r_{i}, r_{i}+g_{i}\right\}$. From [2] we have the

Theorem 1. For $\tau \in \Lambda_{r}^{+}$:

(a) $s(\tau) \in \Lambda_{r}^{++}$

(b) $s(s(\tau))=s(\tau)$,

(c) $P(s(\tau))=P(\tau)$,

and

(d) $s(\tau)=\tau \Leftrightarrow \tau \in \Lambda_{r}^{++} \Leftrightarrow \Gamma(\tau) \in \mathbf{Z}_{+}^{n}$.

Next we define the $i$-blocks of $E_{+}(\tau)$. Let $\tau \in \Lambda_{r}^{+}$. Fix an index $i, 1 \leq$ $i \leq n$. For $j \in \mathbf{Z}_{+}$we define the $j$ th $i$-block of $E_{+}(\tau)$, denoted $Q_{i}(j)$, or $Q_{i(\tau)}(j)$, to be the set of those expressions of $\tau$ as sums of positive roots in $E_{+}(\tau)$ which have $j$ as the coefficient of the simple root $\alpha_{i}=\beta_{i}$. The $i$-blocks partition $E_{+}(\tau)$ for $1 \leq i \leq n$. Also $P(\tau)=\sum_{j \in \mathbf{Z}_{+}}\left|Q_{i}(j)\right| . Q_{i}(j)$ is empty if $j>r_{i}$, where $\Upsilon(\tau)=\left(r_{1}, \ldots, r_{n}\right)$. So $Q_{i}\left(r_{i}\right)$ is the highest occurring $i$-block. 
The lowest occurring one is determined by $\Gamma(\tau)=\left(g_{1}, \ldots, g_{n}\right)$. If $g_{i} \geq 0$, then $Q_{i}(0)$ is nonempty, and the $i$ blocks $Q_{i}(0), Q_{i}(1), \ldots, Q_{i}\left(r_{i}\right)$ are all nonempty. On the other hand if $g_{i}<0$, then $Q_{i}(0), Q_{i}(1), \ldots, Q_{i}\left(-g_{i}-1\right)$ are all empty, and $E_{+}(\tau)$ is comprised of the $i$-blocks from $Q_{i}\left(-g_{i}\right)$ up to $Q_{i}\left(r_{i}\right)$. From [2] we have the

Theorem 2. Let $\tau \in \Lambda_{r}^{+}$with $\Upsilon(\tau)=\left(r_{1}, \ldots, r_{n}\right)$ and $\Omega(\tau)=\left(w_{1}, \ldots, w_{n}\right)$. Let $j \in \mathbf{Z}_{+}$and $i, 1 \leq i \leq n$, be such that $j \leq r_{i}$ and $j \leq w_{i}$. Then, for $i$-blocks in $E_{+}(\tau),\left|Q_{i}(j)\right|=\left|Q_{i}\left(w_{i}-j\right)\right|$.

This theorem is our main inductive tool.

\section{3. $i$-INDUCTIVE WEIGHT SPACES}

We work in the Kostant cone in this section. Let $\tau \in \Lambda_{r}^{++}, \tau \neq 0$, with $\Omega(\tau)=\left(w_{1}, \ldots, w_{n}\right)$ and $\Gamma(\tau)=\left(g_{1}, \ldots, g_{n}\right)$. Then $\tau \in \Lambda_{r}^{++}$implies that $\Gamma(\tau) \in \mathbf{Z}_{+}^{n}$, and so for each $i, 1 \leq i \leq n$, we have $\left|Q_{i}(0)\right|>0$. Then the duality Theorem 2 will yield a nontrivial inductive result, namely that $\left|Q_{i}(0)\right|=$ $\left|Q_{i}\left(w_{i}\right)\right|$, whenever the index $i$ is such that $w_{i}>0$.

However, if $w_{i} \leq 0$ for all $i, 1 \leq i \leq n$, then $\tau=\sum w_{i} \lambda_{i}$ implies that $\tau \in \Lambda_{r}-\Lambda_{r}^{+}$, or else $\tau=0$, because, for irreducible $R$, each fundamental dominant weight $\lambda_{j} \in D$ is such that $\lambda_{j}=\sum_{k=1}^{n} q_{j k} \alpha_{k}$, where each $q_{j k}$ is a rational number and all $q_{j k} \geq 0$. See, for instance, Humphreys [5, Table 1, Section 13.2], or Bourbaki's tables in [1]. So there is at least one $i, 1 \leq i \leq n$, such that $w_{i}>0$.

For $\tau \in \Lambda_{r}^{++}$, with $\Omega(\tau)=\left(w_{1}, \ldots, w_{n}\right)$, we say $\tau$ is $i$-inductive if $w_{i}>0$ for the index $i, 1 \leq i \leq n$. If $\tau$ is $i$-inductive for all $i, 1 \leq i \leq n$, then $\tau$ is inductive, i.e., as a weight $\tau$ is strongly dominant, or in the dominant chamber determined by $B$. Then we have shown

Proposition 3. Let $\tau \in \Lambda_{r}^{++}$. Then $\tau$ is $i$-inductive for at least one index $i$, $1 \leq i \leq n$, or else $\tau=0$.

\section{4. $i$-BLOCKS AND THE PARTITION FUNCTION}

Let $\tau \in \Lambda_{r}^{+}$with $\Upsilon(\tau)=\left(r_{1}, \ldots, r_{n}\right)$. Fix $i, 1 \leq i \leq n$. Let $k, j \in \mathbf{Z}_{+}$ such that $k \leq j \leq r_{i}$. Let $\theta \in Q_{i}(j)=Q_{i(\tau)}(j)$ in $E_{+}(\tau)$. Then $\theta$ is a sum of positive roots adding up to $\tau$ as a sum of simple roots, with the $\alpha_{i}=\beta_{i}$ coefficient exactly $j$. If we let $\theta_{1}=\theta-k \beta_{i}$ denote the same sum of positive roots, except the $\alpha_{i}=\beta_{i}$ coefficient is now $j-k$, then we have that $\theta_{1} \in$ $Q_{i\left(\tau-k \alpha_{i}\right)}(j-k)$. If we had started with $\theta_{1}$ and then added $k$ to the $\alpha_{i}=\beta_{i}$ coefficient etc., then we obtain the following

Proposition 4. Let $\tau \in \Lambda_{r}^{+}$with $\Upsilon(\tau)=\left(r_{1}, \ldots, r_{n}\right)$. Let $k, j \in \mathbf{Z}_{+}$such that $k \leq j \leq r_{i}$, where $1 \leq i \leq n$. Then, computing in $E_{+}(\tau)$ and in $E_{+}\left(\tau-k \alpha_{i}\right)$, we obtain $\left|Q_{i(\tau)}(j)\right|=\left|Q_{i\left(\tau-k \alpha_{i}\right)}(j-k)\right|$. 
Now suppose that $\tau \in \Lambda_{r}^{++}$with $\Upsilon(\tau)=\left(r_{1}, \ldots, r_{n}\right)$ and $\Gamma(\tau)=$ $\left(g_{1}, \ldots, g_{n}\right)$. Then $\Gamma(\tau) \in \mathbf{Z}_{+}^{n}$, and so for each $i, 1 \leq i \leq n$, we have that $\left|Q_{i}(j)\right|>0$ for $0 \leq j \leq r_{i}$. Thus, for each $i$,

$$
P(\tau)=\sum_{j=0}^{r_{i}}\left|Q_{i}(j)\right|,
$$

where $Q_{i}(j)=Q_{i(\tau)}(j)$. Also for each $i$, if $k \in \mathbf{Z}_{+}, k \leq r_{i}$, then, by Proposition 4,

$$
P\left(\tau-k \alpha_{i}\right)=\sum_{j=k}^{r_{i}}\left|Q_{i}(j)\right|,
$$

where $Q_{i}(j)=Q_{i(\tau)}(j)$ again, i.e., we are still computing $i$-blocks in $E_{+}(\tau)$. In particular, for each $i, 1 \leq i \leq n$, subtracting equation 2 from equation 1 when $k=1$ we obtain

$$
\left|Q_{i}(0)\right|=P(\tau)-P\left(\tau-\alpha_{i}\right),
$$

where $Q_{i}(0)=Q_{i(\tau)}(0)$ and $r_{i} \geq 1$. Inductively, if $k \leq r_{i}$, recalling that $P(\theta)=0$ for $\theta \in \Lambda_{r}-\Lambda_{r}^{+}$, we have

$$
\left|Q_{i}(k)\right|=P\left(\tau-k \alpha_{i}\right)-P\left(\tau-(k+1) \alpha_{i}\right),
$$

where $Q_{i}(k)=Q_{i(\tau)}(k)$.

\section{MAIN THEOREM}

We begin in the Kostant cone. Let $\tau \in \Lambda_{r}^{++}$with $\tau \neq 0$ and $\Upsilon(\tau)=$ $\left(r_{1}, \ldots, r_{n}\right), \Omega(\tau)=\left(w_{1}, \ldots, w_{n}\right)$, and $\Gamma(\tau)=\left(g_{1}, \ldots, g_{n}\right)$. Then, by Proposition $3, \tau$ is $i$-inductive for at least one $i, 1 \leq i \leq n$. Fix one such in$\operatorname{dex} i$. Then $w_{i}>0, w_{i}=r_{i}-g_{i}, g_{i} \geq 0$ because $\tau$ is in the Kostant cone, and so $r_{i} \geq 1$. Then, by equation $3,\left|Q_{i}(0)\right|=P(\tau)-P\left(\tau-\alpha_{i}\right)$, where $Q_{i}(0)=Q_{i(\tau)}(0)$. By the duality Theorem 2 we have $\left|Q_{i}(0)\right|=\left|Q_{i}\left(w_{i}\right)\right|$, but $w_{i}=r_{i}-g_{i}$, where $g_{i} \geq 0$, so $w_{i} \leq r_{i}$, and so, by equation 4 , we have that $\left|Q_{i}\left(w_{i}\right)\right|=P\left(\tau-w_{i} \alpha_{i}\right)-P\left(\tau-\left(w_{i}+1\right) \alpha_{i}\right)$, where $Q_{i}\left(w_{i}\right)=Q_{i(\tau)}\left(w_{i}\right)$. Thus $P(\tau)-P\left(\tau-\alpha_{i}\right)=P\left(\tau-w_{i} \alpha_{i}\right)-P\left(\tau-\left(w_{i}+1\right) \alpha_{i}\right)$. Further $P(\tau)>P\left(\tau-\alpha_{i}\right)$, by equation 3 , because $\left|Q_{i}(0)\right|>0$, and in fact $P\left(\tau-\alpha_{i}\right) \geq P\left(\tau-w_{i} a_{i}\right)$, with equality only when $w_{i}=1$, because of equation 2 and the fact that none of the $i$-blocks in equation 1 are empty. Thus we have

Theorem 5. Let $\tau \in \Lambda_{r}^{++}, \tau \neq 0$, with $\Omega(\tau)=\left(w_{1}, \ldots, w_{n}\right)$. There is at least one index $i, 1 \leq i \leq n$, such that $w_{i} \geq 1$, and

$$
P(\tau)-P\left(\tau-\alpha_{i}\right)=P\left(\tau-w_{i} \alpha_{i}\right)-P\left(\tau-\left(w_{i}+1\right) \alpha_{i}\right),
$$

and such that $P(\tau)>P\left(\tau-\alpha_{i}\right) \geq P\left(\tau-w_{i} \alpha_{i}\right)>P\left(\tau-\left(w_{i}+1\right) \alpha_{i}\right)$, with $P\left(\tau-\alpha_{i}\right)=P\left(\tau-w_{i} \alpha_{i}\right) \Leftrightarrow w_{i}=1$. 
In a more general setting let $\theta \in \Lambda_{r}^{+}$. Let $\tau=s(\theta)$. Then $P(\theta)=P(s(\theta))=$ $P(\tau)$ and $\tau$ is in the Kostant cone. Either $\tau=0$, in which case $P(\tau)=1$, or we apply Theorem 5 to inductively compute $P(\tau)=P(\theta)$.

\section{EXAMPLES}

If $R$ is of type $A_{2}$, then the Kostant cone, $\Lambda_{r}^{++}$, is just the set of $r \alpha_{1}+r \alpha_{2}$ for $r \in \mathbf{Z}_{+}$, a ray inside the closure of the dominant chamber in $\Lambda$ with respect to $B$. If $\tau \neq 0$ and $\tau \in \Lambda_{r}^{++}$, then $\Upsilon(\tau)=(r, r), \Gamma(\tau)=(0,0)$, and $\Omega(\tau)=(r, r)$. So each nonzero member of the Kostant cone is inductive. In fact, for $i=1$ or 2 , with $\tau \in \Lambda_{r}^{++}, \tau \neq 0$,

$$
P(\tau)=P\left(\tau-\alpha_{i}\right)+1
$$

because $\tau-w_{i} \alpha_{i}=\tau-r \alpha_{i}=r \alpha_{j}$, where $i \neq j$, and $P\left(r \alpha_{j}\right)=1$, while $P\left(\tau-\left(w_{i}+1\right) \alpha_{i}\right)=P\left(-\alpha_{i}+r \alpha_{j}\right)=0$.

If $R$ is of type $C_{2}$, with largest root $2 \alpha_{1}+\alpha_{2}$, then $\Lambda_{r}^{++}$coincides with the closure of the dominant chamber in $\Lambda$, i.e., for $\tau \in \Lambda_{r}^{+}$with $\Upsilon(\tau)=\left(r_{1}, r_{2}\right)$ we obtain $\Gamma(\tau)=\left(2 r_{2}-r_{1}, r_{1}-r_{2}\right)$ and $\Omega(\tau)=\left(2 r_{1}-2 r_{2}, 2 r_{2}-r_{1}\right)$. So, if $\Gamma(\tau) \in \mathbf{Z}_{+}^{2}$ we have $\Omega(\tau) \in \mathbf{Z}_{+}^{2}$ and vice versa. Then $\tau \in \Lambda_{r}^{++}$is inductive unless $r_{1}=2 r_{2}$ or $r_{1}=r_{2}$, i.e., unless $\tau$ is on the boundary of the Kostant cone, see [2]. On the boundary $\tau$ is only 1- or 2-inductive, not both.

If $R$ is of type $G_{2}$, with largest root $3 \alpha_{1}+2 \alpha_{2}$, then $\Lambda_{r}^{++}$properly contains the closure of the dominant chamber of $\Lambda$, i.e., if $\tau \in \Lambda_{r}^{+}$with $\Upsilon(\tau)=\left(r_{1}, r_{2}\right)$, then we have $\Gamma(\tau)=\left(3 r_{2}-r_{1}, r_{1}-r_{2}\right)$ and $\Omega(\tau)=\left(2 r_{1}-3 r_{2}, 2 r_{2}-r_{1}\right)$. So $\tau \in \Lambda_{r}^{++}$when $3 r_{2} \geq r_{1} \geq r_{2}$, but $\tau$ is only inductive when $2 r_{2}>r_{1}>\left(\frac{3}{2}\right) r_{2}$, and is otherwise only 1 - or 2-inductive, not both.

\section{REFERENCES}

1. N. Bourbaki, Groupes et algebres de Lie, Chap. IV, V, VI, Hermann, Paris, 1968.

2. R. Deckhart, The Kostant cone and the combinatorial structure of the root lattice, J. Alg., (to appear).

3. __ Modular maximal vectors and the Kostant cone, J. Alg., to appear.

4. __ , An modular maximal vectors, I: Bases, rank 2 families, and an introduction to products, Comm. Alg., (to appear).

5. J. E. Humphreys, Introduction to Lie algebras and representation theory, Springer-Verlag, Berlin, Heidelberg, and New York, 1972.

6. __ Ordinary and modular representations of Chevalley groups, Springer-Verlag, Berlin, Heidelberg, and New York, 1976.

7. R. V. Moody and J. Patera, Fast recursion formula for weight multiplicities, Bull. Amer. Math. Soc. (New Series) 7 (1982), 237-242.

8. __ Characters of elements of finite orders in Lie groups, SIAM J. Alg. Disc. Meth. 5 (1984), 359-383.

9. A. Pianzola, On the regularity and rationality of certain elements of finite order in Lie groups, J. Reine Angew. Math. 377 (1987), 40-48. 